\title{
Time-resolved plasma measurements in Ge-doped silica exposed to infrared femtosecond laser
}

\author{
M. Lancry, ${ }^{1, *}$ N. Groothoff, ${ }^{2}$ B. Poumellec, ${ }^{1}$ S. Guizard, ${ }^{3}$ N. Fedorov,${ }^{3}$ and J. Canning ${ }^{2}$ \\ ${ }^{1}$ LPCES/ICMMO, UMR CNRS-UPS 8182, Université Paris Sud 11, Bâtiment 410, 91405 Orsay, France \\ ${ }^{2}$ Interdisciplinary Photonics Laboratories, School of Chemistry, The University of Sydney, 206 NIC, ATP, Eveleigh, NSW, 1340 Australia \\ ${ }^{3}$ Laboratoire des Solides Irradiés/CEA IRAMIS, Ecole Polytechnique, Palaiseau, France
}

(Received 29 June 2011; revised manuscript received 15 November 2011; published 8 December 2011)

\begin{abstract}
Using a time-resolved interferometric technique, we study the laser-induced carrier-trapping dynamics in $\mathrm{SiO}_{2}$ and $\mathrm{Ge}$-doped $\mathrm{SiO}_{2}$. The fast trapping of electrons in the band gap is associated with the formation of self-trapped excitons (STE). The STE trapping is doping dependent in $\mathrm{SiO}_{2}$. The mean trapping time of electrons excited in the conduction band was found to be significantly lower in Ge-doped silica (75 $\pm 5 \mathrm{fs}$ ) when compared to pure silica ( $155 \pm 5 \mathrm{fs}$ ). At our concentration level, this indicates that the plasma properties are determined by the presence of easily ionizable states such as the presence of Ge atoms in the glass network. Therefore, we suggest that in Ge-doped silica there exist an additional trapping pathway that leads to a significantly faster excitons trapping and a higher plasma density when compared to undoped silica.
\end{abstract}

DOI: 10.1103/PhysRevB.84.245103

PACS number(s): 78.47.J-, 52.25.Mq, 81.05.Kf, 82.53.-k

\section{INTRODUCTION}

A single processing tool is the manufacturer's dream for fabricating various components on a common substrate, enabling complete integration into functional and compact systems. Femtosecond lasers are seen as a viable contender for such a tool, at least at the design phase. Today advanced femtosecond laser systems offer a myriad of material interactions in silica-based glasses, from surface machining, to annealing, forming, and refractive index changes (isotropic or anisotropic) writing. ${ }^{1,2}$ Recently, other properties have also arisen, including chirality, ${ }^{3,4}$ directional dependent writing, ${ }^{3-6}$ glass decomposition, ${ }^{7}$ nanocluster precipitation and shaping, ${ }^{8}$ and elemental distribution with a subwavelength resolution. ${ }^{7,9}$ No other technique has the potential to realize 3D multicomponent photonic devices fabricated in one single step within a variety of transparent materials. These interactions potentially enable the development of a new generation of powerful, complex components for micro-optics, telecommunications, optical data storage, imaging, biophotonic, and much more. ${ }^{10,11}$

From the fundamental point of view, the development of femtosecond laser systems has prompted the investigation of many nonlinear physical phenomena, such as multiphotoninduced absorption, plasma formation, and avalanche ionization in glasses. Indeed, in the case of a multiphoton absorption (MPA), it is possible to achieve electronic interband transitions [from the valence band (VB) to the conduction band (CB)]. Specifically, for 800-nm infrared (IR) laser and pure silica glass it needs 5-6 photons. ${ }^{12,13}$ In such a case, multiphoton ionization (MPI) leads to free electron. Another process that leads to band-to-band transition is tunneling ionization produced by the distortion of the band structure under the electromagnetic field. Both processes are in competition, as described in Refs. 14 and 15 . Once the free-electron density becomes nonzero, MPA-inducing high energy electrons lead to electron-electron collision processes, which increase further the free-electron density, creating plasma. Although point defects caused by such intense irradiation have been identified in fluorescence, electron spin resonance (ESR), and other studies, ${ }^{16-18}$ the mechanism of formation of induced modifications in glass is not yet understood.
In an attempt to further resolve these matters, time-resolved experiments using frequency-based spectral interferometry have been performed in dielectric media and especially in silica. ${ }^{12,19,20}$ This method is very powerful because it allows direct in situ observation of the modification of the dielectric function of the material due to the pump pulse in the first instant of the irradiation. From this, the lifetime of photoexcited carriers is measured, ${ }^{20}$ and the electron-plasma density is extracted. Thus, in this paper, spectral interferometry is used to probe the temporal dynamics of a system perturbed by a single pump pulse. In particular the change of the electronicexcitation density has been compared in this way in two silica-based glasses: pure vitreous silica $\left(\mathrm{SiO}_{2}\right)$ and germaniadoped silica $\left(\mathrm{GeO}_{2}: \mathrm{SiO}_{2}\right)$, using various laser intensities from $1 \mathrm{TW} / \mathrm{cm}^{2}$ up to $60 \mathrm{TW} / \mathrm{cm}^{2}$. In Ge-doped silica we would expect that the presence of easily ionizable states [for example, oxygen-deficient centers' (ODC) defects or Ge(1), i.e., a trapped electron on a fourfold-coordinated Ge] would impact laser-induced plasma kinetics. ${ }^{21,22}$

\section{EXPERIMENTS}

In the experiment reported here, a Ti:Sapphire chirpedpulse amplified laser, with a pulse duration $\tau \sim 60 \mathrm{fs}$, wavelength $\lambda_{p}=800 \mathrm{~nm}$, and a repetition rate $=20 \mathrm{~Hz}$, were used. A shutter controlled by a computer selected individual pulses. A lens with focal length $f=20 \mathrm{~cm}$, corresponding to a numerical aperture, $N A=0.02$, was used to focus the pump laser beam. Notice that the high peak power of the laser pulses induces nonlinear propagation effects (e.g., self-focusing) that strongly distort the spatial and temporal profile of the laser pulse in a manner that is difficult to predict. ${ }^{19}$ In the following we will thus roughly estimate the equivalent intensity in air. We estimate that the equivalent laser intensities in air range from $1 \mathrm{TW} / \mathrm{cm}^{2}$ up to $60 \mathrm{TW} / \mathrm{cm}^{2}$.

The samples were pieces of $10 \times 10 \times 0.1 \mathrm{~mm}^{3}$ synthetic silica (Suprasil Type I) or $4.9 \mathrm{w} \%$ Ge-doped silica with optical quality $(<\lambda / 10)$ polished surfaces. They were translated perpendicularly to the propagation direction of the pump and the probe beams to avoid multiple interactions, i.e., we realized 
single-shot measurements. The interaction region was onto the sample front face.

The frequency-based spectral interferometry technique involves two low energy probe pulses, separated in time by a delay $\tau$, that is large compared to their duration and analyzed in a spectrometer. A schematic diagram of the experimental setup of a time-resolved interferometric measurement is shown in Ref. 20. The laser-probe beam was split into two identical ("twin pulses") probe pulses and recombined together using a Michelson interferometer. The probe beams pass through an optical delay line and are transmitted through the dielectric sample. The geometry is neither collinear nor transverse to the pump; the average angle is $\theta \sim 16^{\circ}$ off the pump-propagation direction. In our case the first pulse probes the system before the pump pulse and acts as a reference pulse. The second pulse probes the system at a finite delay $\tau$, after the pump pulse. The perturbation, induced by the pump pulse, leads to a change $\Delta \Phi(t)$, of the relative phase between the twin pulses. This phase shift $\Delta \Phi$, results in a distortion of the fringes in the interference spectrum of the stretched (through a monochromator) twin pulses, and the fringe contrast (proportional to transmittance) varies accordingly. Spectral interferometry uses this distortion to measure $\Delta \Phi$, which is a measure in the change in dielectric constant real part. The initial interferogram is acquired without any pump pulse to serve as reference. A second interferogram is measured while an intense pump pulse excites the dielectric between the reference and probe pulse. In this configuration the phase shift $\Delta \Phi(t)$ is given by: $\Delta \phi(t)=\frac{2 \pi L}{\lambda} \Delta n(t)$, where $\lambda=\frac{2 \pi c}{\omega}$ is the probe-beam wavelength, $L$ is the length over which the probe and the pump beams overlap within the sample ( $\approx 80 \mu \mathrm{m}$ in this experiment), and $\Delta n(t)$ is the instantaneous change in the real part of refractive index that results from the pump-induced excitation. Note that by using the contrast of the fringes, spectral interferometry can also be used to extract the change in absorption coefficient (i.e., the change in the imaginary part of the refractive index).

Notice that the plasma density is not homogeneous both in the propagation direction and the radial direction. ${ }^{12,19}$ We have thus chosen to probe an area without noticeable propagation effect. In addition it should be noted that this effect impacts our measurements in the same manner whatever doping may be (at our concentration level) since the nonlinear index $n_{2}$ does not change significantly. Our results can thus be compared. The reproducibility has been also checked.

\section{RESULTS}

\section{A. $\mathrm{SiO}_{2}$ glass}

In Fig. 1 the black full-line curve corresponds to the measured phase shifts at $800 \mathrm{~nm}$ at $300 \mathrm{~K}$ in a $\mathrm{SiO}_{2}$ sample for fixed-pump intensity around $15 \mathrm{TW} / \mathrm{cm}^{2}$. There are several possible origins for the corresponding refractive-index change after photo-irradiation of valence electrons in a glass matrix. ${ }^{20}$ The first part of the curves contributes positively (analogous to a convex lens) to the phase shift because the nonlinear index is positive at the probe wavelength. It is observed in all materials and will occur as long as the pump and the probe pulses overlap in time within the sample. The delay corresponding to the maximum value of this term has been used to define the zero of our

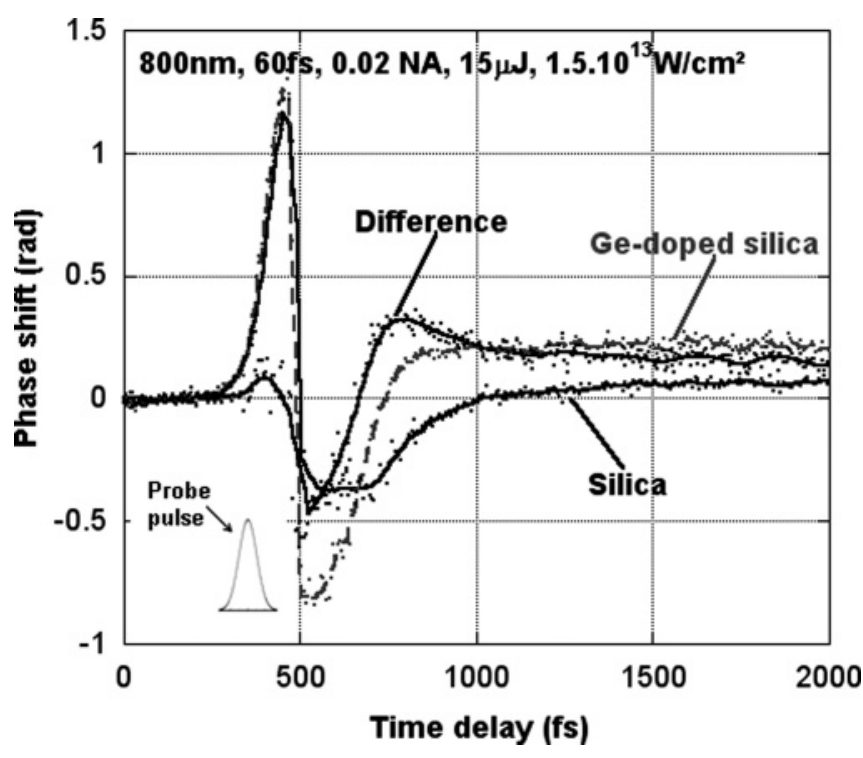

FIG. 1. Phase shift as function of time delay measured in $\mathrm{SiO}_{2}$ and Ge-doped $\mathrm{SiO}_{2}$ for the same pump intensity $15 \mu \mathrm{J}$ (or $15 \mathrm{TW} / \mathrm{cm}^{2}$ in our experimental conditions). The indicated curve is for the difference between those two samples. The probe wavelength is $800 \mathrm{~nm}$, and the sample temperature is $300 \mathrm{~K}$. For sake of comparison, we add the probe-pulse shape in free space, i.e., $60 \mathrm{fs}$ pulse duration. The full lines correspond to guides for eye.

time delay curves. Notice that the full width at half maximum (FWHM) is much larger than expected from the convolution of the probe and the pump-pulse duration $(\approx 60 \mathrm{fs})$. This temporal broadening (estimated to $85 \pm 5 \mathrm{fs}$ ) is likely due to the groupvelocity dispersion in the optics during the beam pathway. The second part is proportional to $N_{\mathrm{CB}}$, the density of electrons in the $\mathrm{CB}$, and is always negative. This term accounts for the observed negative phase shift shown in the curves. The last part stands for the density of trapped electrons $N_{\mathrm{tr}}$. Its sign is determined by the relative energy values of $\omega_{\mathrm{tr}}$ (the excitation energy of the trapping site) and $\omega$. For the experiment described here, the observation of a positive phase shift at the end of the curves indicates that there is trapping of electrons. This means that the transient absorption band $\omega_{\text {tr }}$ associated with the trapping site corresponds to higher frequency than the probe-beam frequency $\left(\omega_{\text {tr }}>\omega\right)$. Consequently the phase shift $\Delta \Phi_{\infty}$ measured at a sufficiently large delay $(\approx 1.5 \mathrm{ps})$ after the laser pulse allows us to calculate the density of electrons that has been previously excited, $N_{\text {tr }}$, in the solid at the end of the laser pulse. ${ }^{12,20}$

\section{B. Ge-doped $\mathrm{SiO}_{2}$ glass}

In Fig. 1 the grey-dotted curve corresponds to the phase shifts measured at $800 \mathrm{~nm}$ in a $4.9 \mathrm{w} \%$ Ge-doped $\mathrm{SiO}_{2}$ sample for fixed-pump intensity around $15 \mathrm{TW} / \mathrm{cm}^{2}$. First we observed a positive phase shift due to the Kerr effect that is not significantly modified when compared to $\mathrm{SiO}_{2}$. This is in agreement with the nonlinear index $n_{2}$ that was found to depend with the Ge content as follows: $n_{2} 10^{-20} \mathrm{~m}^{2} / \mathrm{W}=2.76$ $+0.0974 . x$, where $x$ is the $\mathrm{GeO}_{2}$ concentration in $\mathrm{mol} \%$. $^{23}$ The change in nonlinear index is thus known to be less than $10 \%$ and is quite negligible. The Kerr effect is immediately followed by a negative phase shift (proportional to $N_{\mathrm{CB}}$ ) that 


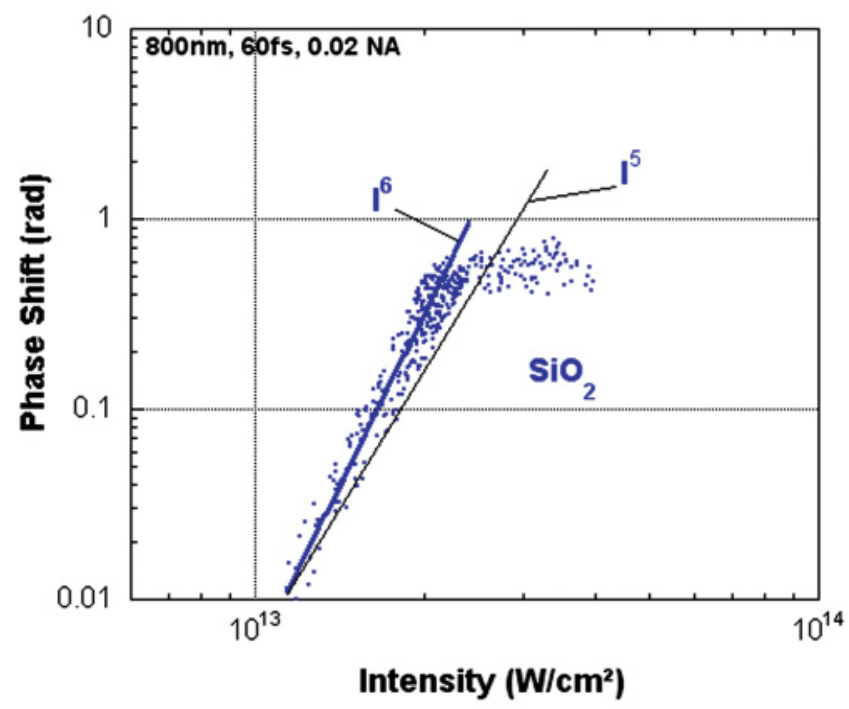

FIG. 2. (Color online) Intensity dependence of the phase shift $\left(\Delta \Phi_{\infty}\right)$ measured in pure $\mathrm{SiO}_{2}$ at a sufficiently large delay $(\Delta t \approx 1.5$ ps) after the pump. The full lines indicate the power laws obtained at low intensity. The probe wavelength is $800 \mathrm{~nm}$, and the sample temperature is $300 \mathrm{~K}$. The pump and the probe polarizations were linearly polarized and parallel to each other. The optical breakdown (OB) threshold is estimated to be around $20 \mathrm{TW} / \mathrm{cm}^{2}$ in these experiments.

is significantly larger than in $\mathrm{SiO}_{2}$. Next, as for pure $\mathrm{SiO}_{2}$, the subsequent evolution of the phase shift toward a positive value indicates trapping of the electrons, but the $\Delta \Phi_{\infty}$ is 3 times higher in Ge-doped silica. In addition it should be noted that the time to get a positive phase shift is significantly smaller in Ge-doped $\mathrm{SiO}_{2}$. This indicates that electrons trapping time $\tau_{\mathrm{tr}}$ is significantly smaller.

Furthermore, if we assume in first approximation that the contributions from $\mathrm{Si}$ and $\mathrm{Ge}$ are just additive, we can note that performing the spectrum difference between the phase shift for pure silica and from Ge-doped silica, and we record the change in the phase shift under doping. This is the full-line curve called difference that is shown in Fig. 1.

\section{Intensity dependence}

Figures 2 and 3 present the change of $\Delta \Phi_{\infty}$ with the incident-peak intensity $I$ at $800 \mathrm{~nm}(\omega=1.55 \mathrm{eV})$ in, respectively, pure $\mathrm{SiO}_{2}$ and $\mathrm{Ge}$-doped $\mathrm{SiO}_{2}$ glasses for $1.5 \mathrm{ps}$ after the pulse maximum. Noticeably, below $20 \mathrm{TW} / \mathrm{cm}^{2}$, $\Delta \Phi_{\infty}$ is observed to vary as $I^{6}$ in $\mathrm{SiO}_{2} \cdot{ }^{12,13}$ In contrast the $\Delta \Phi_{\infty}$ is observed to vary as $I^{5}$ in Ge-doped $\mathrm{SiO}_{2}$ (as shown in Fig. 3). In addition the efficiency is significantly higher in Ge-doped $\mathrm{SiO}_{2}$. Finally, above $20 \mathrm{TW} / \mathrm{cm}^{2}$, we can observe a saturation effect. Notice that if this density is not too high, $\Delta \Phi_{\infty}$ is directly proportional to $N_{\text {tr }}\left(\Delta \Phi_{\infty} \propto N_{\text {tr }}\right) .{ }^{20}$

\section{DISCUSSION}

\section{A. Laser intensity dependence (Figs. 2 and 3)}

\section{Slope change}

At first, it is likely that the slope's behavior is caused by the order of the nonlinear process responsible for the injection

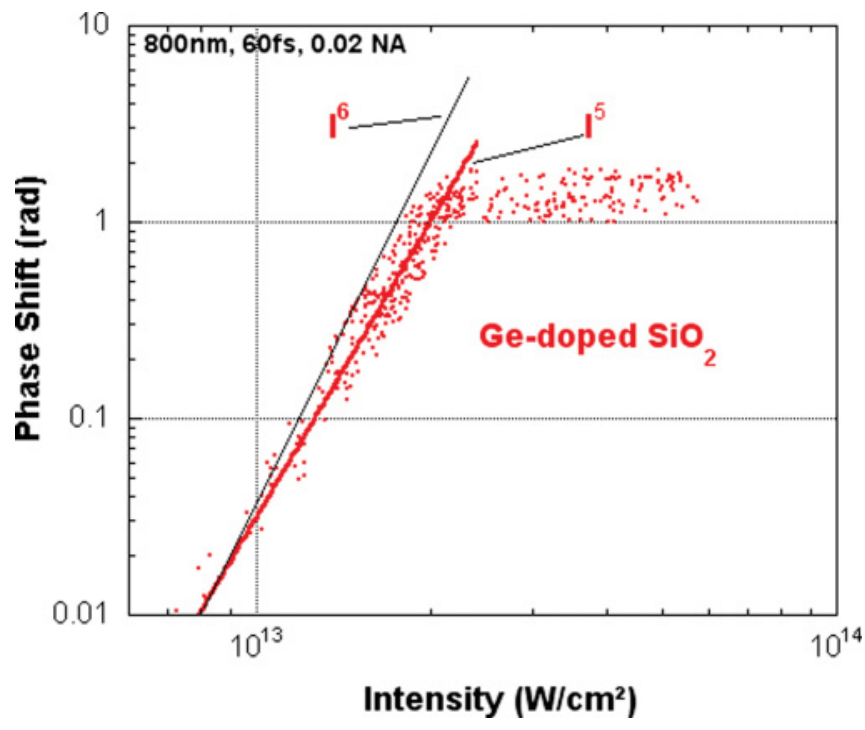

FIG. 3. (Color online) Intensity dependence of the phase shift $\left(\Delta \Phi_{\infty}\right)$ measured in Ge-doped $\mathrm{SiO}_{2}$ at a sufficiently large delay ( $\Delta t \approx 1.5 \mathrm{ps}$ ) after the pump pulse. The full lines indicate the power laws obtained at low intensity. The probe wavelength is $800 \mathrm{~nm}$, and the sample temperature is $300 \mathrm{~K}$. The pump and the probe polarizations were linearly polarized and parallel to each other. The optical breakdown (OB) threshold is estimated to be around $20 \mathrm{TW} / \mathrm{cm}^{2}$ in these experiments.

of valence electrons in the lowest CB. Indeed, in the MPI regime, the rate is $\sigma_{k} I^{k} \rho_{a t}$, where $\sigma_{k}$ is the MPA coefficient for absorption of $k$ photons and $\rho_{a t}$ is the density at the top of the VB: i.e., $2.2 \times 10^{22} / \mathrm{cm}^{3}$ and $N_{\text {tr }} \sim \sigma I N_{0}$. The number of photons required is determined by the smallest $k$, which satisfies the relation $k \cdot h \omega>E_{g}$ (forbidden gap of the dielectrics). At low intensity $\left[<20 \mathrm{TW} / \mathrm{cm}^{2}\right.$ in $\mathrm{SiO}_{2}$; i.e., below the optical breakdown (OB) threshold, ${ }^{24} \Delta \Phi_{\infty}$ is shown to vary as $I^{6}$ in $\mathrm{SiO}_{2}$, so we concluded an underpinning six photon absorption process in agreement with Refs. 12 and 13, which is consistent with the fact that the bandgap in $\mathrm{SiO}_{2}$ is equal to $9 \mathrm{eV}\left(6 \omega=9.3 \mathrm{eV} E_{\mathrm{g}}\left(\mathrm{SiO}_{2}\right) \approx 9 \mathrm{eV}\right)$. This strongly indicates that the dominant excitation process in this intensity range is MPI at least up to the OB threshold because there is another possibility with lower nonlinearity. Indeed, depending on the laser wavelength and intensity, there are two different regimes of photo-ionization: MPI and tunneling ionization that scales more weakly with the laser intensity.

In the same manner we can likely consider a five photon absorption process in $4.9 \mathrm{w} \%$ Ge-doped $\mathrm{SiO}_{2}$. This is in agreement with literature where the bandgap of strongly Ge-doped glass is "shown" to be around $7.1 \mathrm{eV}^{25}(5 \omega=$ $\left.7.75 \mathrm{eV}>E_{g}\left(\mathrm{Ge}: \mathrm{SiO}_{2}\right) \approx 7.1 \mathrm{eV}\right)$. In fact this apparent absorption edge in the Ge-doped $\mathrm{SiO}_{2}$ is determined in part by preexisting absorption of defects' centers such as GeODC(I) that absorb strongly around $7.6 \mathrm{eV}^{26}$ The presence of additional near-edge absorption extends the apparent absorption edge tail and decreases MPI slope. In addition Smelser et al. ${ }^{27}$ have reported that the initial slope of the permanent refractive-index change writing kinetics in Ge-doped optical fibers follows an $I^{5}$ evolution with the laser intensity indicating a five photons absorption process. Then, due to a higher MPA coefficient 
$\left(\sigma_{5}=1.810^{-55} \mathrm{~s}^{-1} \mathrm{~cm}^{10} \mathrm{~W}^{-5}\right)$ for Ge-doped silica $\left(\sigma_{6}=\right.$ $4.510^{-69} \mathrm{~s}^{-1} \mathrm{~cm}^{12} \mathrm{~W}^{-6}$ in $\left.\mathrm{SiO}_{2}\right)$, this leads to a slightly higher excitation density $\left(\sigma_{5} I^{5}\right.$ is two times higher than $\left.\sigma_{6} I^{6}\right)$ when compared to silica, ${ }^{21}$ at least for "low" laser intensity (below $60 \mathrm{TW} / \mathrm{cm}^{2}$ ), which is in agreement with our observations. ${ }^{1}$

\section{Saturation effect}

Above the OB threshold (defined previously), we observe, in agreement with Refs. 12 and 13, a saturation effect that could suggest a change in the ionization regime. Indeed, depending on the laser wavelength and intensity, there are two different regimes of photo-ionization: MPI and tunneling ionization that scales more weakly with the laser intensity. The transition between MPI and tunneling ionization was expressed by Keldysh. ${ }^{15}$ When the so-called Keldysh parameter $\gamma$ is greater than 1.5 (for "low" intensity), photo-ionization is a MPI, whereas when $\gamma<1.5$ (high intensity) we are in tunneling regime. This is well modeled in Ref. 14. In addition, at this intensity range, the occurrence of electronic avalanche, due to strong heating of the conduction electrons, cannot be excluded. Stuart et $a .^{28}$ developed a model of avalanche ionization in which the avalanche rate depends linearly on the laser intensity (i.e., $\eta=\alpha I$, where $\alpha$ is the avalanche-ionization coefficient). On the other hand Thornber et al. ${ }^{29}$ predict an avalanche rate that depends on the square root of the laser intensity. However, playing with the pulse duration in order to clarify the role of avalanche ionization, we have found no evidence of avalanche in previous work, ${ }^{12}$ and these results have been confirmed be more recent experiments. ${ }^{13}$

In principle we could try to use our plasma measurements' dependence with the laser intensity to distinguish between the two ionization mechanisms. However, we would like to point out that the experimentally determined carrier densities are only correct under the assumption of a homogeneous excitation profile inside the sample (along the propagation direction). Detailed investigation of carrier density and beam propagation at intensities above and below the OB threshold ${ }^{12,13}$ showed that for short pulses the critical plasma density $\left(\sim 1.7 .10^{21} / \mathrm{cm}^{3}\right.$ at $800 \mathrm{~nm}$ ) can be generated during the beginning of the pulse, leading to both strong absorption and reflectivity of the pump pulse. In this regime the excitation density keeps increasing with intensity only within a thinner layer of material and this leads, thus, to saturation effect elsewhere. Therefore, we conclude that under our experimental conditions the spatial averaging totally masks possibility of more localized high intensities.

To summarize, above $20 \mathrm{TW} / \mathrm{cm}^{2}$ (OB threshold in silica) the occurrence of tunneling or avalanche ionization cannot be excluded. Indeed, as already mentioned, for short pulses and around the OB threshold the excitation density is strongly inhomogeneous, decreasing by almost two orders of magnitude in a few microns. Since the dephasing of the probe pulse is integrated over several $\mu \mathrm{m}$, a change in the ionization mechanism-from multiphoton to tunneling, for instance-occurring within the head of the laser track (i.e., where the intensity is the highest) cannot be excluded.

\section{B. Trapping kinetics: a comparison between silica and Ge-doped silica}

For the sake of clarity, we will present here an approximate expression that estimates the phase shift $\Delta \phi$, within an order of magnitude ${ }^{20}$ and which is more convenient to identify the contribution of each effect-a principle of summation of contributions is assumed. It is based on the following mechanism simplification:

$$
\begin{aligned}
& \text { during the pulse } \mid \begin{array}{l}
X \stackrel{\text { MPI or tunnel }}{\longrightarrow} X^{+}+e_{C B}^{l E} \\
e_{C B}^{l E} \stackrel{\mathrm{MPA}}{\longrightarrow} e_{C B}^{h E}
\end{array} \\
& e_{C B}^{h E}+e_{V B} \stackrel{\text { avalanche or forest fire }}{\longrightarrow} 2 e_{C B}^{l E} \\
& \text { after the pulse } \mid \begin{array}{l}
e_{C B}^{l E}+X^{+} \stackrel{\text { electronic trapping in a few } 100 \mathrm{fs}}{\longrightarrow} \mathrm{STE} \\
\mathrm{STE} \stackrel{\tau_{a n}>n s}{\longrightarrow} \mid \begin{array}{l}
X+h \nu_{1} \\
\text { defects }
\end{array}
\end{array},
\end{aligned}
$$

where $l E$ and $h E$ correspond to electrons near the bottom of the $\mathrm{CB}$ and more excited electrons, respectively; $\mathrm{VB}$ is for valence band; $X$ is a regular site of glass near the oxygen atoms; and $X^{+}$is the corresponding self-trapped hole (STh), i.e., an electron and a hole in interaction. Self-trapped excitons (STE) are formed in less than one ps. Indeed, it is now well known that ionizing radiation produces $\mathrm{STE}$ in $\mathrm{SiO}_{2} \cdot{ }^{30-34}$ In $\mathrm{SiO}_{2}$, besides radiative recombination, they may relax into $\mathrm{SiE}^{\prime}$ and $\mathrm{NBOHC},{ }^{19}$ labeled previously as defects. The production of permanents' defects is less than $1 \%$ of the regular relaxation. ${ }^{17}$ However, since both would be formed a few ns after the excitation and probe pulses, they are not seen in the present experiment since measurements are taken during the first picoseconds. The experiment therefore neglects the actual possibility for STE to generate defects such as SiODC that can potentially act as further electron sources and $\mathrm{Si}-\mathrm{ODC}^{+}$centers as trapping sites. We have checked their contribution; however, by performing the measurement after $2 \times 10^{4}$ pulses, these contributions were not detected. Further, we did not detect any difference between Infrasil or Suprasil silica, ${ }^{35}$ which indicates that if these defects are generated they play a negligible role at this concentration level.

In the kinetics scheme the density of excitations and the Coulomb force between electron and hole, along with the absence of sufficient existing traps and $e$ donors in silica, leads to consider that they are interdependent and hence can be considered as a single species. Then the scheme can be further reduced to

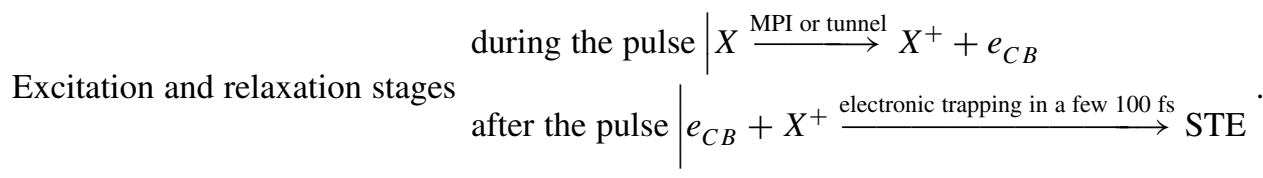


In the following we call $N_{0}$ the background atom density $\left(2.2 .10^{22} / \mathrm{cm}^{3}\right.$ in silica), $N_{\mathrm{CB}}$ is the concentration of electronhole pairs, $N_{\mathrm{tr}}$ is the concentration of STE, and we note with $\sigma_{6}$ the 6 photons' absorption cross-section (MPI contribution) over a trapping time $\tau$. Then, based on the previously mentioned mechanism, we get the following overall rate equation:

$$
\frac{d N_{C B}}{d t}=N_{0} \sigma_{6} I(t)^{6}-\frac{N_{C B}}{\tau} \frac{d N_{t r}}{d t}=\frac{N_{C B}}{\tau} .
$$

These equations are one-order equations or exponential kinetics. The first equation can be solved easily, and it gives $N_{C B}(t)=\mathrm{N}_{0} \sigma_{6} \exp \left(-\frac{t}{\tau}\right) \int_{0}^{t} I\left(t^{\prime}\right)^{6} \exp \left(-\frac{t^{\prime}}{\tau}\right) d t^{\prime}$. Then, the second equation can be solved in turn, and we get $N_{t r}(t)=$ $\frac{1}{\tau} \int_{0}^{t} N_{C B}\left(t^{\prime}\right) d t^{\prime}$. We note that $N_{\mathrm{CB}}$ can be seen as a broadening of the $I(t)^{6}$ function on the long-time side, whereas $N_{\text {tr }}$ is the total number of electrons that has been excited.

Therefore considering the scheme for pure silica, we can speculate the following for Ge-doped silica:

$$
\begin{aligned}
& \text { during the pulse } \mid \begin{array}{l}
X(\mathrm{Si}) \stackrel{\text { MPI or tunnel }}{\longrightarrow} X(S i)^{+}+e_{B C} \\
X(G e) \stackrel{\text { MPI or tunnel }}{\longrightarrow} X(G e)^{+}+e_{B C}
\end{array} \\
& \text { after the pulse } \mid \begin{array}{l}
e_{C B}+X(S i)^{+} \stackrel{\text { electronic trapping in a few } 100 \text { fs }}{\longrightarrow} \operatorname{STE}(S i) \\
e_{C B}+X(G e)^{+} \stackrel{\text { electronic trapping in a few } 100 \text { fs }}{\longrightarrow} \operatorname{STE}(G e)
\end{array}
\end{aligned}
$$

where $X(\mathrm{Si})$ and $X(\mathrm{Ge})$ means sites around oxygen near $\mathrm{Si}$ and $\mathrm{Ge}$, respectively. The reaction constant of the second equation is probably larger than the first one as the excitation of an electron from VB to Ge 4s-p orbitals needs only 5 photons, but, on the contrary, the $X(\mathrm{Ge})$ density is 10 times smaller than $X(\mathrm{Si})$ density. On the other hand the trapping rate on a $X(\mathrm{Ge})$ containing hole is probably faster, as shown later. As the excitation density is not large enough to consider two excitations at the same location, electron-hole pairs can be considered again as preserved entities. So, we can speculate that the kinetics around $\mathrm{Si}$ or around $\mathrm{Ge}$ are independent. Calling $N_{\mathrm{CBSi}}, N_{\mathrm{CBGe}}, N_{\mathrm{trSi}}$, and $N_{\text {trGe }}$ the concentration of the different entities, we get simply

$$
\begin{aligned}
& \frac{d N_{C B S i}}{d t}=N_{0} \sigma_{6} I(t)^{6}-\frac{N_{C B S i}}{\tau_{S i}} \frac{d N_{t r S i}}{d t}=\frac{N_{B C S i}}{\tau_{S i}} \\
& \frac{d N_{C B G e}}{d t}=N_{0} \sigma_{5} I(t)^{5}-\frac{N_{C B G e}}{\tau_{G e}} \frac{d N_{t r G e}}{d t}=\frac{N_{B C G e}}{\tau_{G e}} .
\end{aligned}
$$

It is worth noticing that $\sigma_{5}$ contains the molar fraction of Ge as the number of electrons that can be potentially excited is restricted to $\mathrm{Ge}$ neighboring. The previously mentioned equations are one-order equations or exponential kinetics again. Those equations have been solved, as previously, for pure silica,

$$
\begin{aligned}
& N_{C B S i}(t)=N_{0} \sigma_{6} \exp \left(-\frac{t}{\tau_{S i}}\right) \int_{0}^{t} I\left(t^{\prime}\right)^{6} \exp \left(\frac{t^{\prime}}{\tau_{S i}}\right) d t^{\prime} \\
& N_{t r S i}(t)=\frac{1}{\tau_{S i}} \int_{0}^{t} N_{C B S i}\left(t^{\prime}\right) d t^{\prime} \\
& N_{C B G e}(t)=N_{0} \sigma_{5} \exp \left(-\frac{t}{\tau_{G e}}\right) \int_{0}^{t} I\left(t^{\prime}\right)^{5} \exp \left(\frac{t^{\prime}}{\tau_{G e}}\right) d t^{\prime} \\
& N_{t r G e}(t)=\frac{1}{\tau_{G e}} \int_{0}^{t} N_{C B G e}\left(t^{\prime}\right) d t^{\prime} .
\end{aligned}
$$

It should be noted that for a given trapping time, if we compare $N_{\mathrm{CB}} / \sigma_{n} I_{0}{ }^{n}$ and $N_{\mathrm{tr}} / \sigma_{n} I_{0}{ }^{n}$, varying $n$, the shape does not change significantly nor the amplitude.

\section{Trapping electrons' density: a comparison between silica and Ge-doped silica}

Now consider each species and their contribution to dielectric constant, index, or phase shift.

The corresponding dielectric function for $\mathrm{CB}$ electrons is ${ }^{20}$

$$
\Delta \varepsilon_{N_{C B}}(\omega)=-\frac{N_{C B} e^{2}}{m^{*} \varepsilon_{0}} \cdot \frac{f_{C B}}{\omega^{2}+i \omega / \tau_{e-p}},
$$

where $1 / \tau_{e-p}$ stands for the electron-phonno coupling, with $f_{\mathrm{CB}}$ being the oscillator strength of the intraband transitions and $m^{*}$ the electrons' effective mass within the CB. The "hole part" of the electron-hole species yield negligible contribution to the dielectric constant as its effective mass is much larger (typically 10 times higher) than the excited electrons. The index contribution is thus

$$
-\frac{e^{2}}{2 n_{0} \varepsilon_{0}} \cdot \frac{N_{C B} f_{C B}}{m^{*} \omega^{2}} .
$$

The corresponding dielectric function for STEs is ${ }^{20}$

$$
\Delta \varepsilon_{N_{t r}}(\omega)=\frac{N_{t r} e^{2}}{m \varepsilon_{0}} \cdot \frac{f_{t r}}{\omega_{t r}^{2}-\omega^{2}-i \omega / \tau_{t r}},
$$

where $1 / \tau_{\text {tr }}$ is the width of STE transition, with $f_{\text {tr }}$ being the oscillator strength of the STE absorption band and $\omega_{\mathrm{tr}}$ the angular frequency of the STE absorption band. The index contribution is thus

$$
-\frac{e^{2}}{2 n_{0} \varepsilon_{0}} \cdot \frac{N_{t r} f_{t r}}{m_{\mathrm{STE}}\left(\omega_{t r}^{2}-\omega^{2}\right)} .
$$

As we look at the changes induced by the light, the normal electrons have not yet been considered specifically, but their polarizability changed under light. It is a nonlinear effect that is translated into Kerr effect. Index contribution is thus $n_{2} . I_{p}(t)$, where $I_{p}(t)$ is the light intensity. This nonlinear-index $n_{2}$-dependence on the Ge content will be as follows: $n_{2}$ $10^{-16} \mathrm{~cm}^{2} / \mathrm{W}=2.76+0.0974 . x$, where $x$ is the $\mathrm{GeO}_{2}$ concentration in $\mathrm{mol} \%{ }^{23}$ 
Finally, collecting all the contributions, the phase shift can be rewritten as the following expression:

$$
\Delta \Phi(t)=\frac{2 \pi}{\lambda} L\left[n_{2} I_{p}(t)+\frac{e^{2}}{2 n_{0} \varepsilon_{0}}\left\{-\frac{N_{C B S i}(t) \cdot f_{C B S i}}{m_{S i}^{*} \omega^{2}}-\frac{N_{C B G e}(t) \cdot f_{C B G e}}{m_{G e}^{*} \omega^{2}}+\frac{N_{t r S i}(t) \cdot f_{t r S i}}{m\left(\omega_{t r S i}^{2}-\omega^{2}\right)}+\frac{N_{t r G e}(t) \cdot f_{t r G e}}{m\left(\omega_{t r G e}^{2}-\omega^{2}\right)}\right\}\right] .
$$

There is a summation rule for an isolated system, such that the sum of all oscillator strength $f_{i}$ for all possible transitions $i$ is equal to unity. To determine a precise value of $f_{i}$ for a particular wavelength would necessitate spectroscopic measurements of the absorption spectrum of excited carriers. Such measurement is unfortunately not available. Thus $f_{\mathrm{CB}}$ is taken equal to 1 for the probe wavelength, which is an overestimated value. We should note anyway that in this model the absolute value of excited carriers can be deduced only if we know all oscillator strengths and all effective masses. Since in the present work we are more interested in trapping kinetics and not the absolute value of excited-carrier densities, this simplifying hypothesis does not have any influence on the final result.

$m^{*}$ is the electron-effective mass in the CB; for low energy electrons $m^{*}$ is close to $0.5 . \mathrm{m}$, assuming a parabolic band. ${ }^{36}$ However it has been shown that for higher electrons' energy, this value increases and follows a trend to saturation around $m$. In addition an averaging is made on the electrons according to their kinetics energy. ${ }^{14}$ In the following we will thus assume that $m^{*}=m$, and we will consider similar values in silica and $\mathrm{Ge}$-doped silica. From the literature an energy of $4.6 \mathrm{eV}$ and $5.6 \mathrm{eV}$ are commonly associated to $\omega_{\mathrm{trSi}}$ for amorphous pure silica. ${ }^{31,34}$ In the case of Ge-doped silica we found a transient absorption band related to a STE at an energy of $4.1 \mathrm{eV}$ associated to $\omega_{\mathrm{trGe}} \cdot{ }^{34}$ Practically, we will consider in each case only the dominant term, i.e., the one which is the closest to resonant transitions for our probe wavelengths. In this model the optical absorption spectra associated with electrons trapped in the band gap are represented by single absorption lines. In other words, $f_{\text {trSi }}$ and $f_{\text {trGe }}$ must also be considered as an adjustable effective parameter because, at least in principle, several transitions with different probabilities are possible. The MPA coefficients $\sigma_{6}$ and $\sigma_{5}$ will be also considered as adjustable parameters but within the data range already reported in the literature. ${ }^{14}$

First, we simulated the $\mathrm{SiO}_{2}$ curve using Eq. (1) with three adjustable parameters $\left(f_{\mathrm{trSi}}, \tau_{\mathrm{trSi}}\right.$, and $\left.\sigma_{6}\right)$ and without the terms related to the presence of Germanium. The fit is shown in Fig. 3 (left side). We found electron-trapping time $\tau_{\text {tr }}$ of $155 \pm 5 \mathrm{fs}$ and an oscillator strength around $0.3 \pm 0.1$, in agreement with previous publication. ${ }^{20}$ Next, we have fixed those parameters, and we simulated the Ge-doped curve using Eq. (1) with "only" three adjustable parameters $\left(f_{\mathrm{trGe}}, \tau_{\mathrm{trGe}}\right.$, and $\left.\sigma_{5}\right)$, as shown in Fig. 4. Finally, we can see the experimental difference and its accurate simulation in Fig. 5, which reproduces quite well the frequency but also the amplitude for long-time delay.

In Table I we present a summary of the set of parameters that fits satisfactorily the measured phase shifts in Fig. 1.

From the simulation (fit of the difference shown in Fig. 5) we obtain thus a density $N_{\text {trSi }}$ around $10^{19} \mathrm{~cm}^{-3}$ close to the $\mathrm{OB}$ threshold in pure silica. As contributions from $\mathrm{Si}$ and $\mathrm{Ge}$

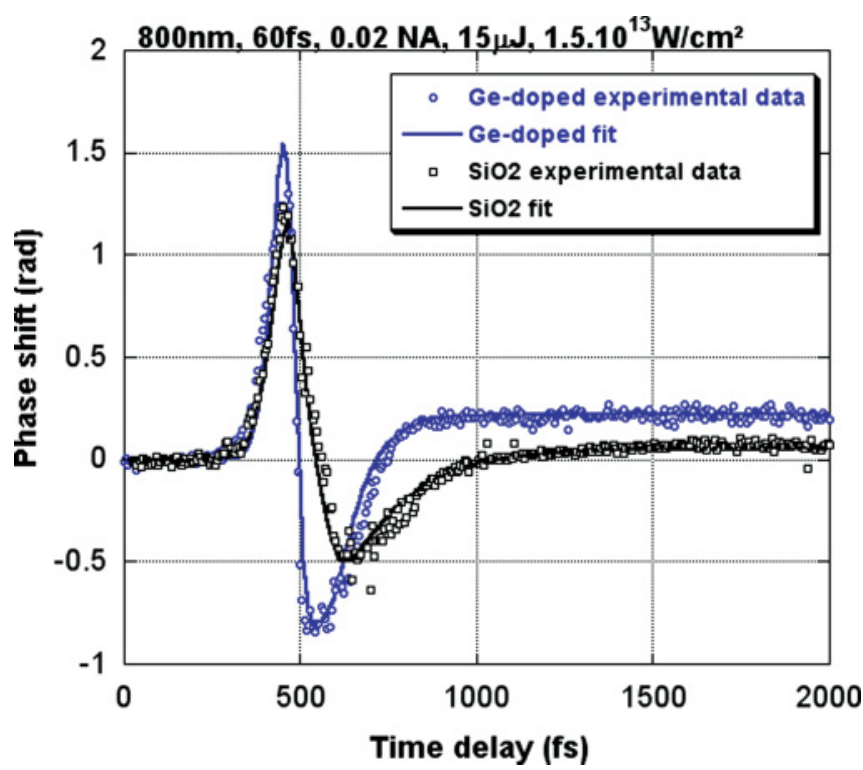

FIG. 4. (Color online) The figure corresponds to the fit (lines) of the experimental data (dots) using the previously mentioned model for $\mathrm{SiO}_{2}$ (in black) and Ge-doped $\mathrm{SiO}_{2}$ samples (in blue/gray) The pump intensity was fixed to $15 \mathrm{TW} / \mathrm{cm}^{2}$ in our experimental conditions.

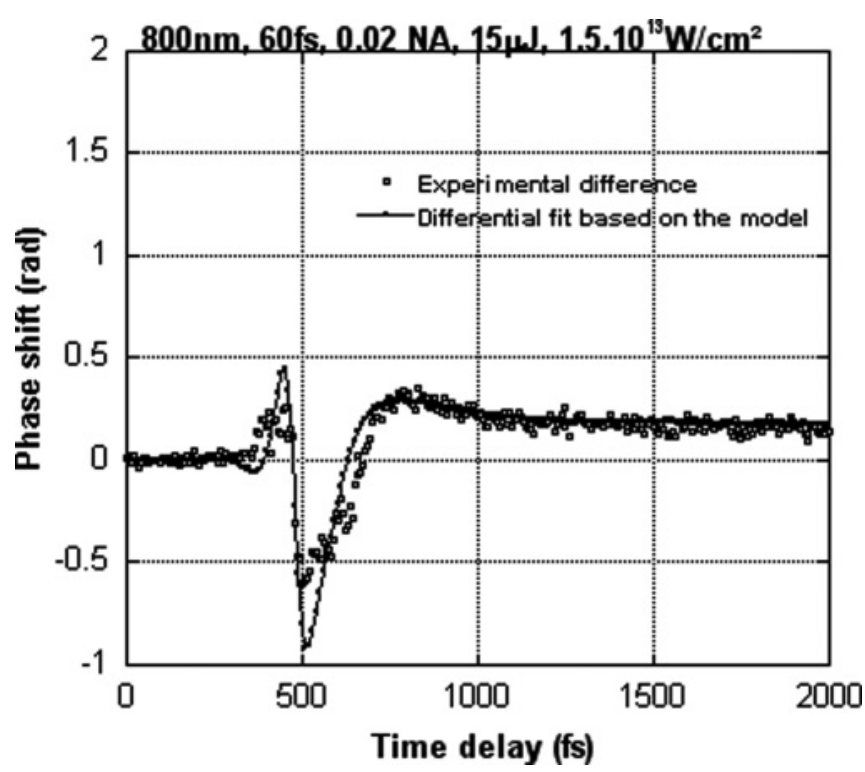

FIG. 5. The figure corresponds to the difference between the $\mathrm{SiO}_{2}$ time-delay curve and the Ge-doped $\mathrm{SiO}_{2}$ time-delay curve; this allows us to highlight the difference between those two samples. The dots are for the experimental difference, and the full line corresponds to differential fit. 
TABLE I. Set of parameters used in the simulation for $\mathrm{SiO}_{2}$ and Ge-doped $\mathrm{SiO}_{2}$.

\begin{tabular}{|c|c|c|c|}
\hline & & $\mathrm{SiO}_{2}$ & Ge-doped $\mathrm{SiO}_{2}(x$ mol\% in $\mathrm{Ge})$ \\
\hline \multicolumn{4}{|l|}{ Fixed parameters } \\
\hline Nonlinear refractive index $\left(\mathrm{cm}^{2} / \mathrm{W}\right)$ & $n_{2}$ & $2.76 .10^{-16}$ & $(2.76+0.097 \cdot x) \cdot 10^{-16}$ \\
\hline Initial valence electron density $\left(\mathrm{cm}^{-3}\right)$ & $N_{0}$ & $2.2 .10^{22}$ & $2.2 .10^{22}$ \\
\hline Order of the multiphoton process & $n$ & 6 & 5 \\
\hline Oscillator strength for the VB-CB transition & $f_{\mathrm{CB}}$ & 1 & 1 \\
\hline Electron effective mass in the CB $(\mathrm{kg})$ & $m^{*}$ & $m_{e}$ & $m_{e}$ \\
\hline Trap level energy (eV) & $\omega_{\mathrm{tr}}$ & 4.6 & 4.1 \\
\hline \multicolumn{4}{|l|}{ Adjustable parameters } \\
\hline Electron trapping time (fs) & $\tau_{\mathrm{tr}}$ & $155 \pm 5$ & $75 \pm 5$ \\
\hline Oscillator strength for the trap level & $f_{\text {tr }}$ & $0.3 \pm 0.1$ & $0.3 \pm 0.1$ \\
\hline Multiphoton cross section & $\sigma$ & $\sigma_{6}=(4.5 \pm 0.2) \cdot 10^{-69} \mathrm{~s}^{-1} \mathrm{~cm}^{12} \mathrm{~W}^{-6}$ & $\sigma_{5}=(1.8 \pm 0.2) \cdot 10^{-55} \mathrm{~s}^{-1} \mathrm{~cm}^{10} \mathrm{~W}^{-5}$ \\
\hline
\end{tabular}

are just additive, we can note that performing the spectrum difference between the phase shift for pure silica and from $\mathrm{Ge}$ doped silica, and we record the change in the phase shift under doping. Furthermore, if we assume in first approximation that silica constants are not changed significantly by the doping, we can deduce the parameters attached to Ge and then compare the concentrations of the different species. Finally we found that the density $N_{\text {trGe }}$ is around $2.2 .10^{19} \mathrm{~cm}^{-3}$ in Ge-doped silica.

From the simulation procedure described previously, we can extract the average electron-trapping time $\tau_{\text {tr }}$. We found $155 \pm 5$ fs in pure silica and only $75 \pm 5$ fs in slightly Ge-doped silica. Surprisingly, doping $\mathrm{SiO}_{2}$ with a few\% of $\mathrm{GeO}_{2}$ leads to a significantly faster electron-trapping time when compared to pure $\mathrm{SiO}_{2}$. An initial idea to explain the faster trapping kinetics could be to think in terms of shorter electron-hole distances in Ge-doped silica, as described in Ref. 20. Knowing exactly the minimum electron-hole distance necessary to prevent the trapping of an electron by a hole is a difficult task, but as a first approximation, one can use the following simple arguments. When an electron-hole pair is created by the pump pulse, there is Coulomb attraction until they are sufficiently far apart. In order to estimate the distance necessary for the carriers to avoid recombination, one can take the distance $\left(r_{c}\right)$ at which the Coulomb energy becomes equal to the thermal energy $\sim 3 / 2 \mathrm{kT}$. One finds $r_{c}\left(\mathrm{SiO}_{2}\right)=95 \AA$ and $r_{c}\left(10 \mathrm{w} \%\right.$ Ge-doped $\left.\mathrm{SiO}_{2}\right)=100 \AA$ for $T=300 \mathrm{~K}$. It can be concluded, therefore, that this simple approach does not explain the observations. We have thus to consider that there is an additional trapping center involving Ge atoms $(\mathrm{Ge} \rightarrow \mathrm{Ge}(1))$. Notice that Ge atoms' density (typically a few $10^{21} / \mathrm{cm}^{3}$ ) is of the same order of magnitude as the typical plasma density induced in our conditions. This additional pathway together with the lower Ge-O bond strength explain the significantly faster trapping when compared to undoped silica.

\section{CONCLUSION}

In summary we have investigated electronic plasma induced by a focused single femtosecond-laser pulse in both synthetic pure silica and Ge-doped silica.

We first measured both density of excitation and the STE trapping kinetic by use of the interference pattern for various laser intensities ranging over (1-60) $\mathrm{TW} / \mathrm{cm}^{2}$. The measured mean value $N_{\mathrm{STE}}$ is of the order of $N_{\mathrm{STE}}$, approximately a few $10^{19} \mathrm{~cm}^{-3}$ in both pure silica and Ge-doped silica. To extract more accurate values, further experiments to probe the plasmadensity distribution along the laser-propagation direction are planned using perpendicular pump-probe geometry.

The mean-trapping time of electrons excited in the $\mathrm{CB}$ was found to be significantly lower in Ge-doped silica $\left(\tau_{\mathrm{tr}} \approx 75 \pm\right.$ $5 \mathrm{fs}$ ) when compared to pure silica ( $\tau_{\mathrm{tr}} \approx 155 \pm 5 \mathrm{fs}$ ). At our concentration level, this indicates that the plasma properties are determined by the presence of easily ionizable states such as the presence of $\mathrm{Ge}$ atoms in the glass network. Therefore, we suggest that in Ge-doped silica there exists an additional trapping pathway that leads to a significantly faster trapping when compared to undoped silica. Future experiments will be dedicated to study the Ge-doping influence from $0.1 \mathrm{w} \%$ up to $30 \mathrm{w} \%$. We expect a significant decrease in the average trapping time due to the additional trapping pathways such as the formation of germanium-electron centers.

The results produced here have provided key insights into possible mechanisms underpinning the irradiation of glass with high-intensity, ultra-short pulses of light. In our experimental conditions MPA is the dominant excitation mechanism up to breakdown threshold, and then a saturation arises due to the formation of a dense plasma, which both strongly absorbs and reflects the remaining laser pulse at the close vicinity of the surface. Thus we do not exclude that tunneling ionization and impact ionization may occur at the highest intensity and within a thin layer beneath the surface. These insights provide a microscopic basis for tailoring and possibly optimizing the processing conditions used to fabricate practical devices in silica and doped silica using such lasers.

\section{ACKNOWLEDGMENTS}

This work has been achieved in the frame of FLAG (Femtosecond Laser Application in Glasses) consortium project with the support of several organisations: the Agence Nationale pour la Recherche (ANR-09-BLAN-0172-01), the RTRA Triangle de la Physique (Réseau Thématique de Recherche Avancée, 2008-056T), the Essonne administrative Department (ASTRE2007), and FP7-PEOPLE-IRSES e-FLAG 247635. 
*matthieu.lancry@u-psud.fr

${ }^{1}$ M. Lancry, B. Poumellec, A. Chahid-Erraji, M. Beresna, and P. Kazansky, Optical Materials Express 1, 711 (2011).

${ }^{2}$ B. Poumellec, M. Lancry, A. Chahid-Erraji, and P. Kazansky, Optical Materials Express 1, 766 (2011).

${ }^{3}$ B. Poumellec, M. Lancry, J. Poulin, and S. Ani-Joseph, Opt. Express 16, 18354 (2008).

${ }^{4}$ B. Poumellec, L. Sudrie, M. Franco, B. Prade, and A. Mysyrowicz, Opt. Express 11, 1070 (2003).

${ }^{5}$ P. Kazansky, W. Yang, E. Bricchi, J. Bovatsek, A. Arai, Y. Shimotsuma, K. Miura, and K. Hirao, Appl. Phys. Lett. 90, 151120 (2007).

${ }^{6}$ B. Poumellec, M. Lancry, J. C. Poulin, and S. Ani-Joseph, Bragg Gratings, Photosensitivity, and Poling in Glass Waveguides, OSA Technical Digest (CD) (Optical Society of America, Quebec, Canada, 2007).

${ }^{7}$ J. Canning, M. Lancry, K. Cook, A. Weickman, F. Brisset, and B. Poumellec, Optical Materials Express 1, 998 (2011).

${ }^{8}$ M. Kaempfe, G. Seifert, K. Berg, H. Hofmeister, and H. Graener, Eur. Phys. J. D 16, 237 (2001).

${ }^{9}$ Y. Shimotsuma, P. Kazansky, J. Qiu, and K. Hirao, Phys. Rev. Lett. 91, 247405 (2003).

${ }^{10}$ K. Itoh, W. Watanabe, S. Nolte, and C. Schaffer, MRS Bull. 31, 620 (2006).

${ }^{11}$ J. Qiu, K. Miura, and K. Hirao, J. Non-Cryst. Solids 354, 1100 (2008).

${ }^{12}$ F. Quéré, S. Guizard, and P. Martin, EPL (Europhys. Lett.) 56, 138 (2001).

${ }^{13}$ V. Temnov, K. Sokolowski-Tinten, P. Zhou, A. El-Khamhawy, and D. von der Linde, Phys. Rev. Lett. 97, 237403 (2006).

${ }^{14}$ A. Couairon, L. Sudrie, M. Franco, B. Prade, and A. Mysyrowicz, Phys. Rev. B 71, 125435 (2005).

${ }^{15}$ L. Keldysh, Sov. Phys. JETP 20, 1018 (1965).

${ }^{16}$ J. Chan, T. Huser, S. Risbud, and D. Krol, Opt. Lett. 26, 1726 (2001).

${ }^{17}$ S. Guizard, P. Martin, G. Petite, P. D'Oliveira, and P. Meynadier, J. Phys. Condens. Matter 8, 1281 (1996).

${ }^{18}$ H. Sun, S. Juodkazis, M. Watanabe, S. Matsuo, H. Misawa, and J. Nishii, J. Phys. Chem. B 104, 3450 (2000).
${ }^{19}$ S. Mao, F. Quéré, S. Guizard, X. Mao, R. Russo, G. Petite, and P. Martin, Appl. Phys. A: Materials Science and Processing 79, 1695 (2004).

${ }^{20}$ P. Martin, S. Guizard, P. Daguzan, G. Petite, P. D'Oliveira, P. Meynadier, and M. Perdrix, Phys. Rev. B 55, 5799 (1997).

${ }^{21}$ M. Lancry, S. Guizard, and B. Poumellec, Femtosecond Laser Microfabrication, OSA Technical Digest (CD) (Optical Society of America, San Jose, California, USA, 2009).

${ }^{22}$ M. Lancry, W. Yang, B. Poumellec, and P. Kazansky, Advances in Optical Materials, OSA Technical Digest (CD) (Optical Society of America, San Jose, California, USA, 2009).

${ }^{23}$ T. Kato, Y. Suetsugu, and M. Nishimura, Opt. Lett. 20, 2279 (1995).

${ }^{24}$ C. Schaffer, A. Brodeur, and E. Mazur, Meas. Sci. Technol. 12, 1784 (2001).

${ }^{25}$ J. Nishii, N. Kitamura, H. Yamanaka, H. Hosono, and H. Kawazoe, Opt. Lett. 20, 1184 (1995).

${ }^{26}$ L. Skuja, J. Non-Cryst. Solids 239, 16 (1998).

${ }^{27}$ C. Smelser, S. Mihailov, and D. Grobnic, Opt. Express 13, 5377 (2005).

${ }^{28}$ B. C. Stuart, M. D. Feit, S. Herman, A. M. Rubenchik, B. W. Shore, and M. D. Perry, Phys. Rev. B 53, 1749 (1996).

${ }^{29}$ K. Thornber, J. Appl. Phys. 52, 279 (1981).

${ }^{30}$ D. L. Griscom, Phys. Rev. B 40, 4224 (1989).

${ }^{31}$ C. Itoh, K. Tanimura, and N. Itoh, J. Phys. C: Solid State Physics 21, 4693 (1988).

${ }^{32}$ C. Itoh, K. Tanimura, N. Itoh, and M. Itoh, Phys. Rev. B 39, 11183 (1989).

${ }^{33}$ N. Itoh, T. Shimizu-Iwayama, and T. Fujita, J. Non-Cryst. Solids 179, 194 (1994).

${ }^{34} \mathrm{~A} . \mathrm{N}$. Trukhin, Excitons, localized states in silicon dioxide and related crystals and glasses, in International School of Solid State Physics, 17th course, NATO Science Series. II Mathematics, Physics and Chemistry, edited by D. Griscom, G. Pacchioni, and L. Skuja, Vol. 2 (2000), pp. 235-283.

${ }^{35}$ M. Lancry, N. Groothoff, S. Guizard, W. Yang, B. Poumellec, P. Kazansky, and J. Canning, J. Non-Cryst. Solids 355, 1057 (2009).

${ }^{36}$ R. Ludeke, E. Cartier, and A. Schenk, Appl. Phys. Lett. 75, 1407 (1999). 